\title{
Evidence of cosmic recurrent and lagged millennia-scale patterns and consequent forecasts: multi-scale responses of solar activity (SA) to planetary gravitational forcing (PGF)
}

\author{
Jorge Sánchez-Sesma \\ Correspondence to: Jorge Sánchez-Sesma (jorgesanchezsesma@yahoo.com)
}

The copyright of individual parts of the supplement might differ from the CC-BY 3.0 licence. 
$1 \quad$ Section S1. Statistical detrending and demodulation of the solar proxy (10Be) record of FN97

$2 \quad 10 \mathrm{Be}$ is a proxy of SA. It is produced by the impacts of galactic cosmic rays on Earth (SS14)

3 in a known process of cosmogenic nucleosynthesis. Cosmic rays are highly energetic charged

4 particles that impact Earth's upper atmosphere and terrain surface, that produce $10 \mathrm{Be}$, and that

5 are modulated by the variation in the strength of the geomagnetic field, as well as by solar

6 magnetic shielding (SS14).

7 In order to homogenize 10Be values, we apply, firstly, a detrending process based on 8 polynomial expressions, and secondly, a demodulation intended to make the variance 9 uniform. Figure S1a shows the polynomial model for detrending, and Figure S1b shows the 10 linear model of the standard deviation for a demodulation. The detrended and demodulated 11 residuals (DDR), of 10Be results are displayed in Figure S1c with two analogue models with 12 lags of 9.6 and 19.2 Kyrs, showing similar variability for the next millennia. Please note in 13 Figure S1c a forecasted decrease of TSI with a Super minimum period from -2 to $-2.5 \mathrm{Kyr}$ BP 14 (from 3950 to 4450 AD). 

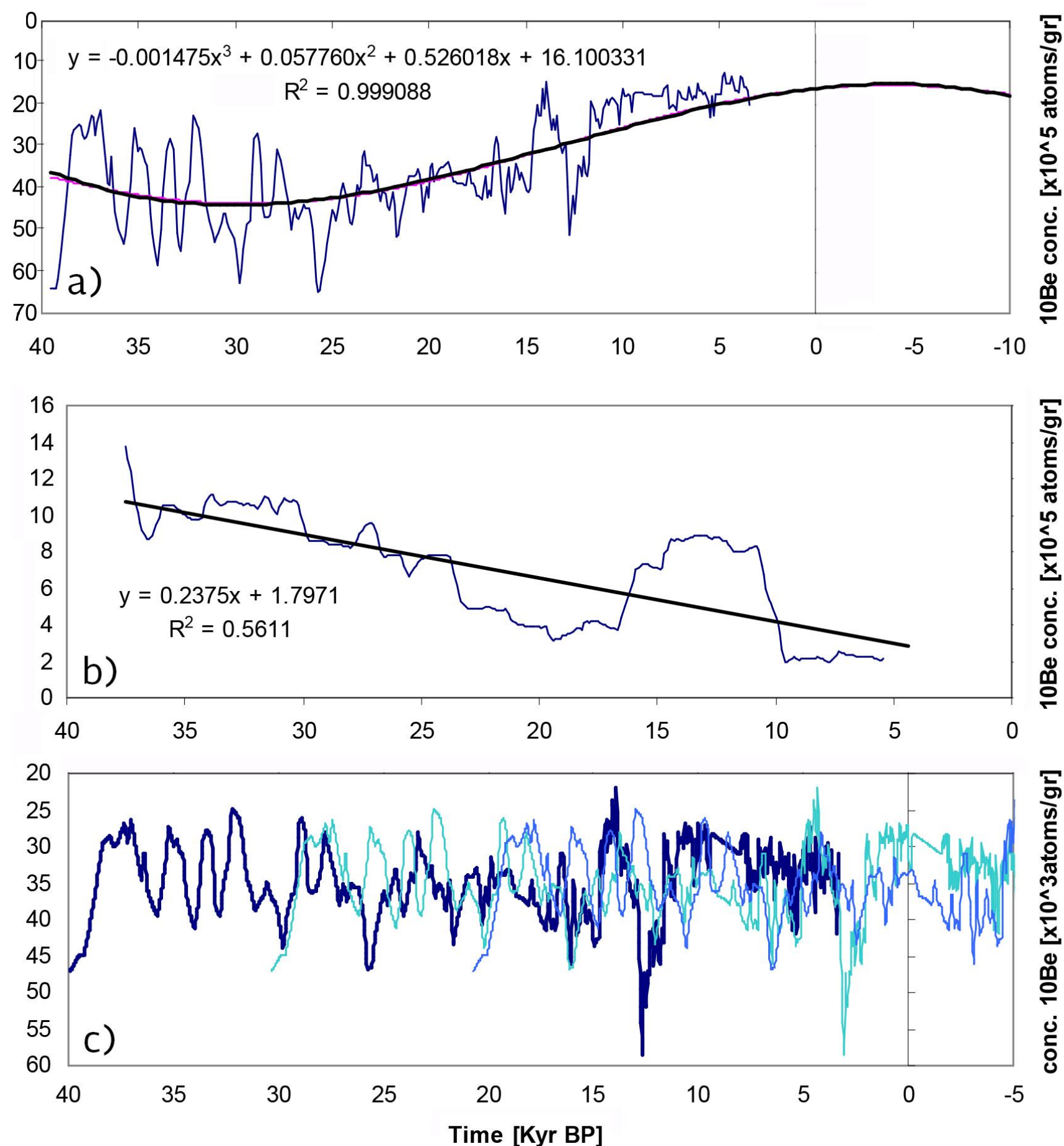

2 Figure S1. GISP2 ice core analysis. a) 10Be concentration [FN97] and a polynomial trend. b) Standard deviation and its linear model. c) Detrended and demodulated residuals (DDR), of 10Be [residual (a)/(linear model of Std. Dev.(b)], and their lagged model for 9.6 and 19.2 Kyrs of this DDR of 10Be are also displayed. Please note that in the following figures: as the 10Be concentration varies inversely with solar activity, TSI, the beryllium scale is inverted, and thus upper parts in this scale indicate high TSI levels. 
1 Section S2. Estimation of a temporal adjustment (bias) of the Greenland 10Be record by SS14.

2 A temporal adjustment for the NEEM 10Be record produced by SS14 is presented. This 3 record is compared with another study with information coming from the NGRIP ice-cores 4 (Kindler et al., 2014), located less than $1000 \mathrm{~km}$ from the NEEM site. A temporal bias 5 correction of 2500 yrs, going back in time, was applied to SS14 data to get the best match 6 with the Kindler et al. (2014) data, and is shown in Figure S2.

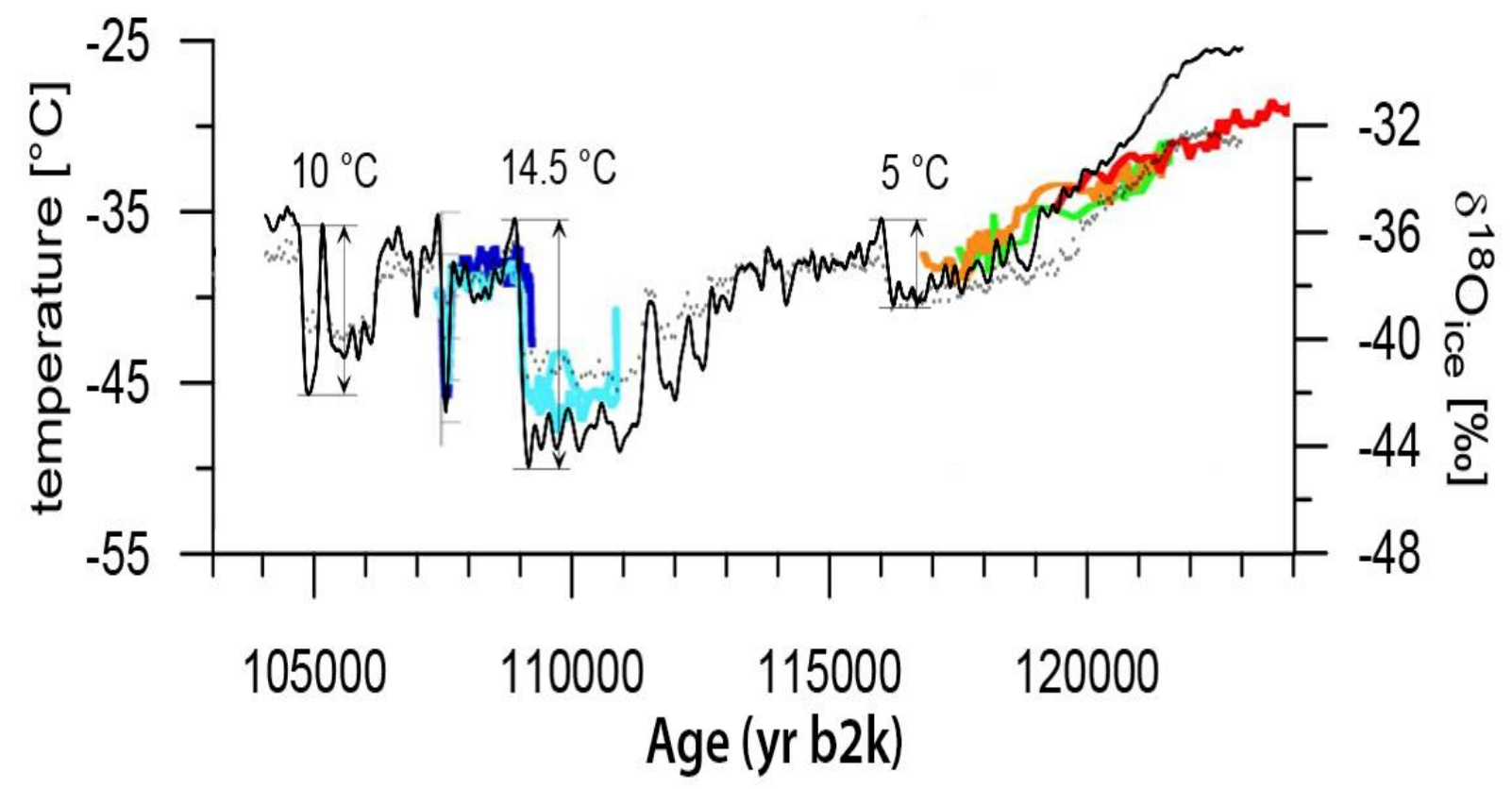

8 Figure S2. Comparison of NGRIP Greenland ice-core reconstructed isotopic anomalies in two different studies

9 (SS14, K14). The gray points indicate anomalies 180 ice, and the black line indicates temperatures from Kindler

10 et al. (2014). Colored lines are anomalies of 180 ice from the SS14 that have been adjusted in time, with a lead 11 of 2500 years to increase the match with K14 results. 
1 Section S3. Detrending and demodulating the solar proxy (10Be) record of FN97, following

2 Alley et al. (1995)

3 Alley et al. (1995) have proposed a method for interpolating between the end-members cases

4 of 10Be wet and dry deposition. They argued that, on average, under conditions of global

5 constant global 10Be production rate and constant dry deposition rate and scavenging ration

6 for precipitation, a linear relation should be expected between 10Be flux and snow flux at

7 GISP2. Then, deviation of measured 10Be from the correlation line can be attributed to

8 changes in the atmospheric concentration of $10 \mathrm{Be}$.

9 Figures S3 and S4 show these processes to obtain the Atmospheric 10Be. Figure S3 shows:

10 a) 10Be concentrations [FN97], b) ice accumulation rates [Alley, 2000], and c) their linear

11 model and correlation. And Figure S4 shows: a) deviations from the linear interpolation

12 (see Fig. S3c), b) their standard deviation, and c) the demodulated deviations with

13 a precessional sinusoidal model.

14 The final atmospheric signal DDR of $10 \mathrm{Be}$ without orbital influences, as suggested by Alley et al. (2000), is displayed in Figure S5 with a simple analogue model with a lag of 9.4 Kyrs, showing a potential decrease of TSI for the next centuries and lower values for the next

17 millennia. Please note in Figure S5 a forecasted decrease of TSI for the next millennia. 


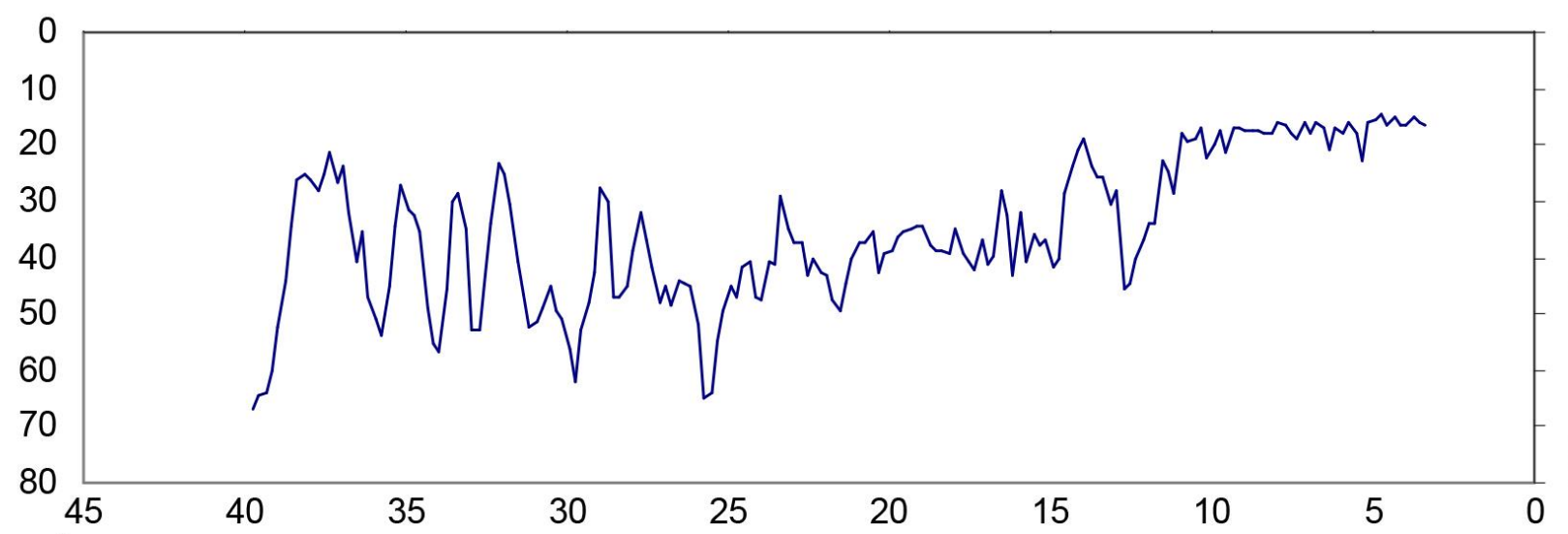

a)

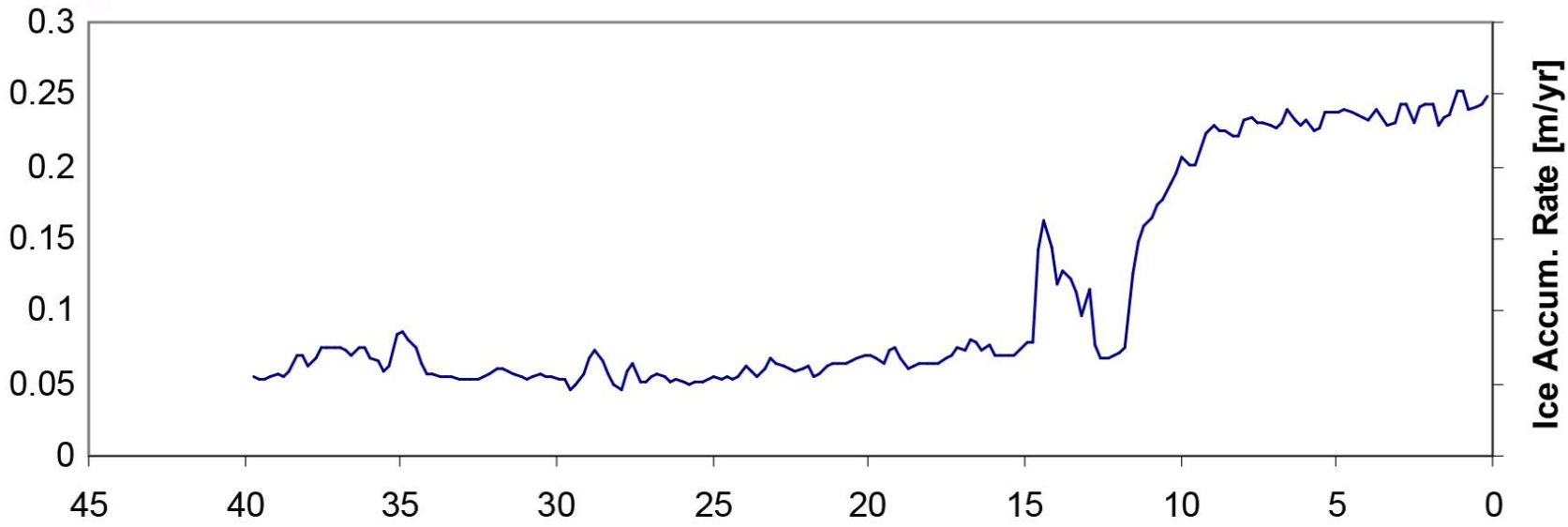

b)

Time [Kyr BP]

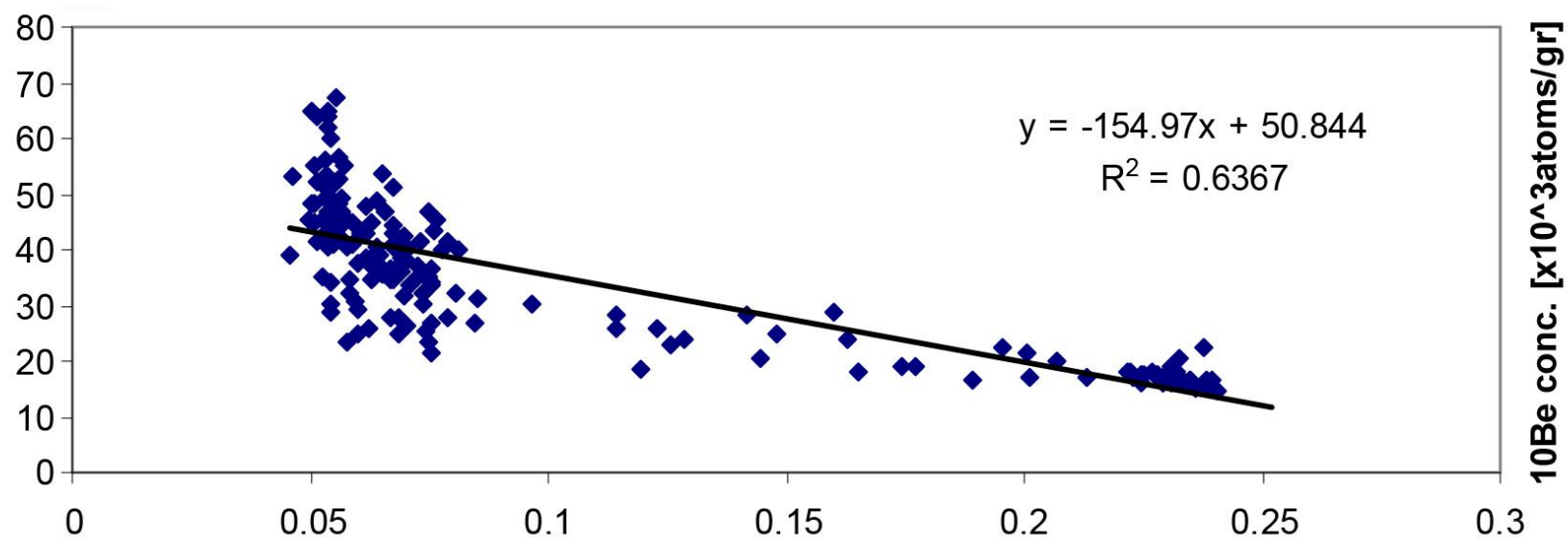

C)

Ice Accum. Rate [m/yr]

2 Figure S3. GISP2 ice core analysis. a) 10Be concentration [FN97], b) ice accumulation rate [Alley, 2000], c)

3 linear correlation model between 10Be concentration and ice accumulation shown in a) and b). 

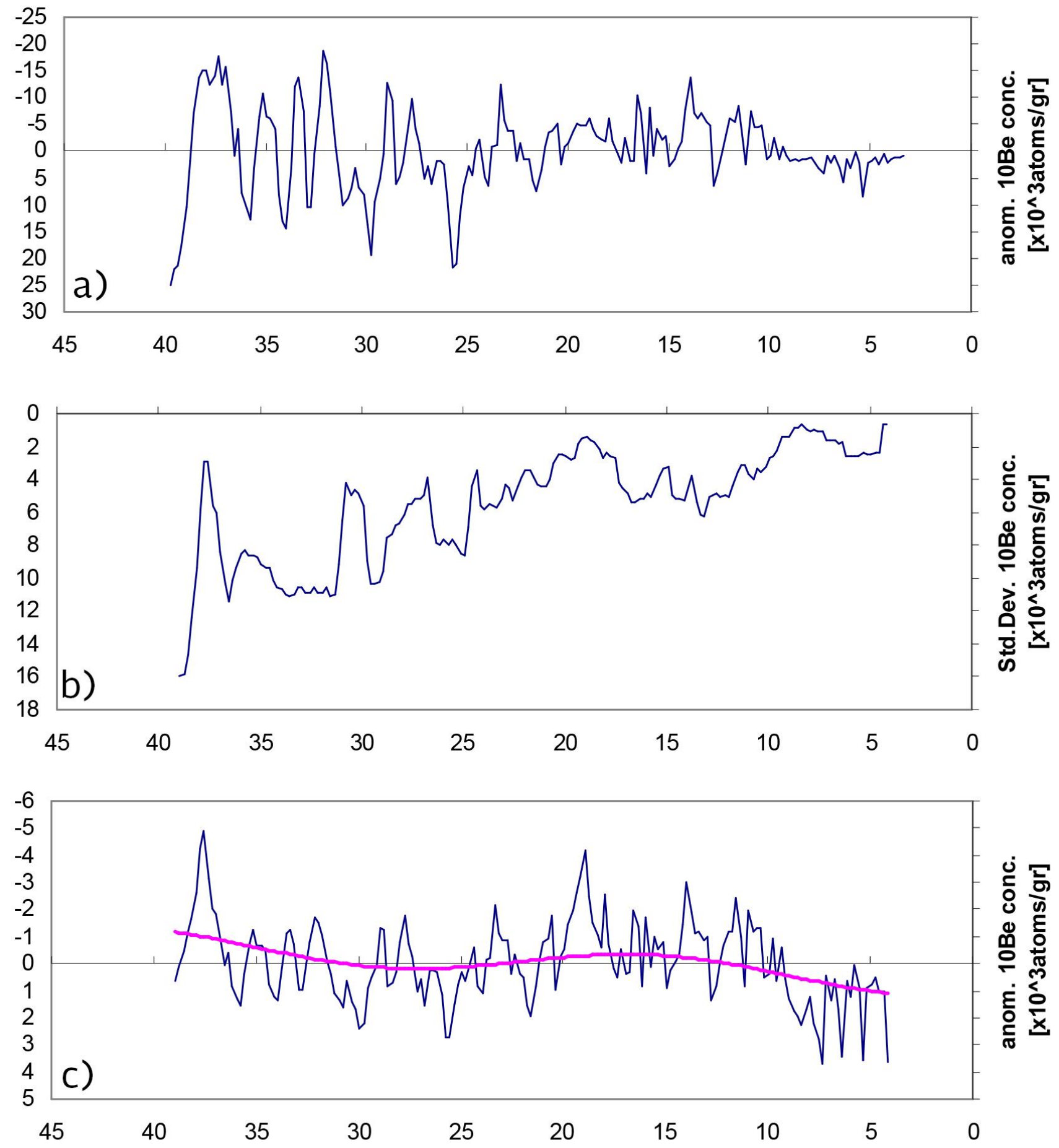

Time [Kyr BP]

2 Figure S4. GISP2 ice core 10Be analysis. a) 10Be residual concentration after eliminating ice accumulation 3 linear contributions (Fig S3c), b) Standard deviation of a), c) Demodulated 10Be [(a)/(b)] with a precession 4 simple linear and sinusoidal model variation for detrending purposes. 


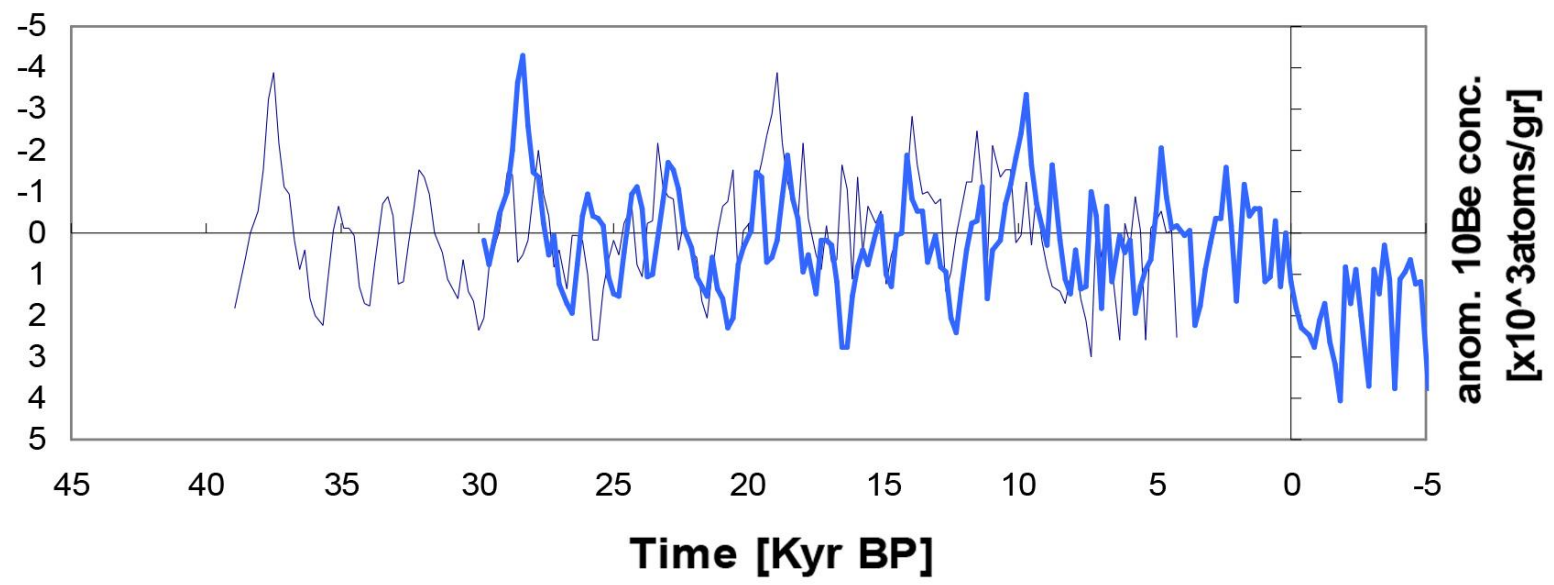

2 Figure S5. GISP2 ice core analysis. 10Be detrended and demodulated residual (DDR) concentration. See Figs. 3 3 and 4. A lagged model (9.4 Kyr) of this 10Be DDR concentration is also displayed.

4

5 


\section{Section S4. Spectral analysis of solar activity}

2 In order to verify the multi-millennia scale solar oscillation of $\sim 9500$ yrs detected in $10 \mathrm{Be}$

3 records, a wavelet analysis was applied to the reconstructed records of TSI, using the online 4 resource by Torrence and Compo (1998).

5 TSI spectral results, displayed in Figure S6, show three main, significant $(\sim 10 \%$ level $)$

6 periodicities around 9000, 5000, and 2400 years. It should be noted that the $S 09$ record also

7 shows relatively high-frequency significant variability with periods around 200 years. 8 


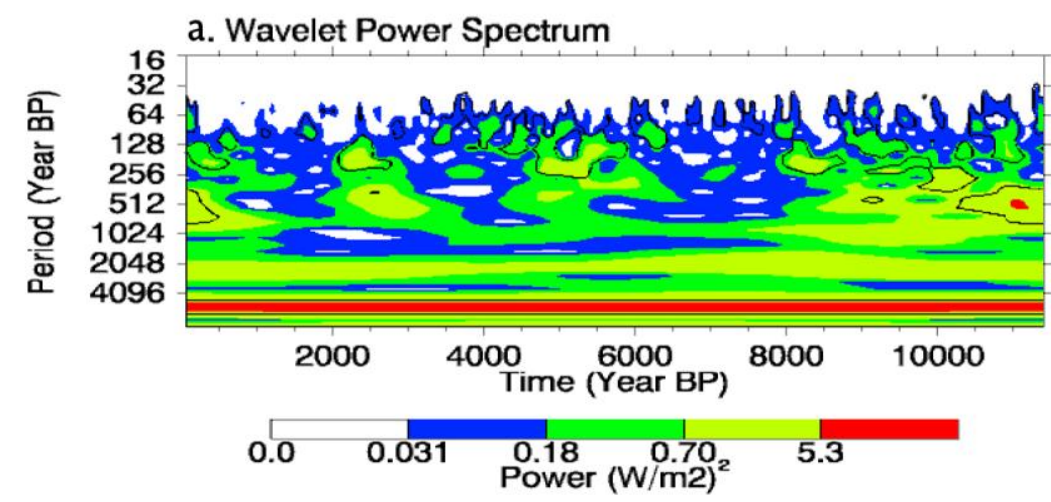

b. Global Wavelet
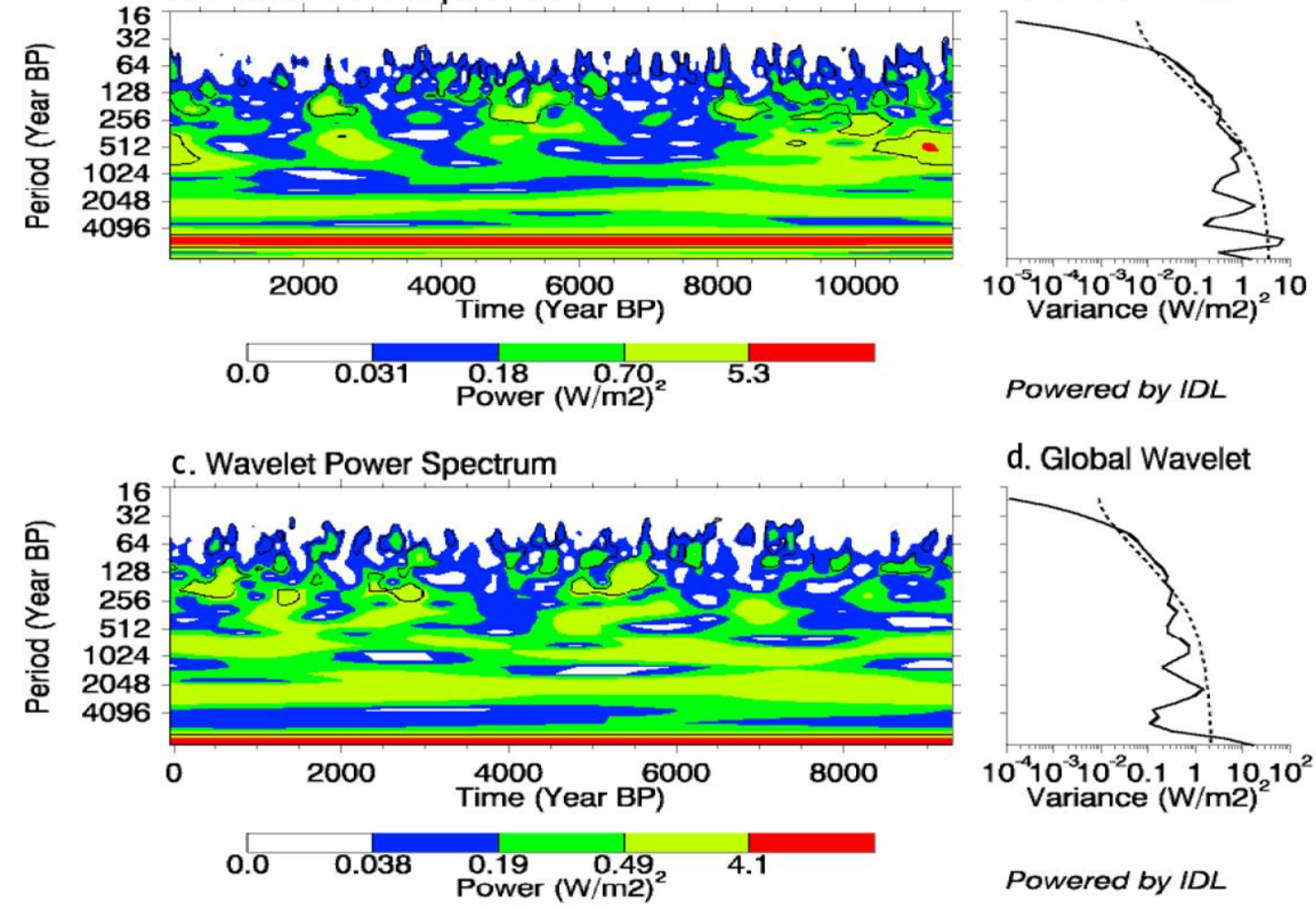

Powered by IDL

d. Global Wavelet

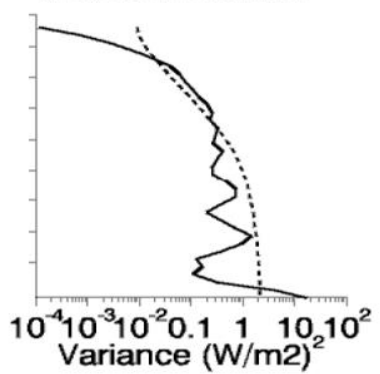

Powered by $I D L$

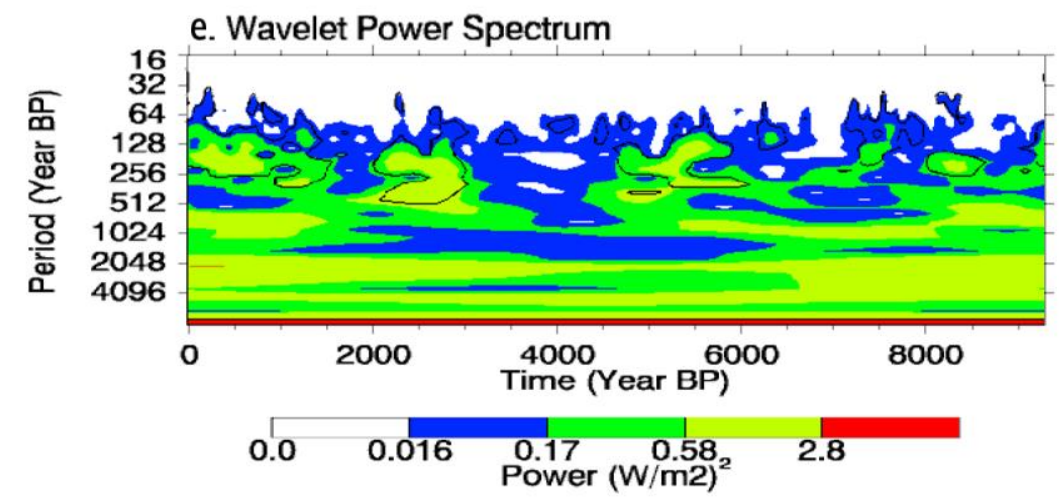

f. Global Wavelet

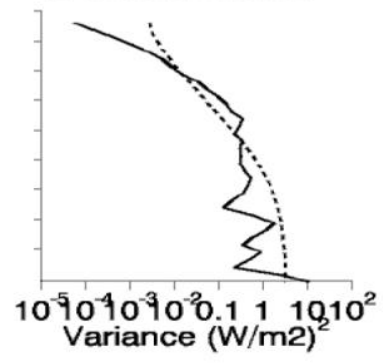

Powered by IDL

Figure S6. (a, c, and e.) The wavelet power spectrum of TSI(S04), TSI(S09), and TSI(S12), respectively. The 3 contour levels are chosen so that $75 \%, 50 \%, 25 \%$, and $5 \%$ of the wavelet power is above each level, 4 respectively. Black contour is the $10 \%$ significance level, using a red-noise (autoregressive lag1) background 5 spectrum. (b, d, and f.) The global wavelet power spectrum (black line). The dashed line is the significance for 6 the global wavelet spectrum, assuming the same significance level and background spectrum as in (a, c, and e, 7 respectively). Reference: Torrence, C. and Compo, G.P., 1998: A Practical Guide to Wavelet Analysis. Bull. 8 Amer. Meteor. Soc., 79, 61-78. 